\title{
CORRIGENDUM
}

\section{Investigation of common, low-frequency and rare genome- wide variation in anorexia nervosa}

LM Huckins, K Hatzikotoulas, L Southam, LM Thornton, J Steinberg, F Aguilera-McKay, J Treasure, U Schmidt, C Gunasinghe, A Romero, C Curtis, D Rhodes, J Moens, G Kalsi, D Dempster, R Leung, A Keohane, R Burghardt, S Ehrlich, J Hebebrand, A Hinney, A Ludolph, E Walton, P Deloukas, A Hofman, A Palotie, P Palta, FJA van Rooij, K Stirrups, R Adan, C Boni, R Cone, G Dedoussis, E van Furth, F Gonidakis, P Gorwood, J Hudson, J Kaprio, M Kas, A Keski-Rahonen, K Kiezebrink, G-P Knudsen, MCT Slof-Op 't Landt, M Maj, AM Monteleone, P Monteleone, AH Raevuori, T Reichborn-Kjennerud, F Tozzi, A Tsitsika, A van Elburg, Eating Disorder Working Group of the Psychiatric Genomics Consortium, DA Collier, PF Sullivan, G Breen, CM Bulik and E Zeggini

Molecular Psychiatry advance online publication, 19 September 2017; doi:10.1038/mp.2017.202

Correction to: Molecular Psychiatry advance online publication, 25 July 2017; doi: 10.1038/mp.2017.88
The fortieth author's name was listed incorrectly. The correct presentation is A Keski-Rahkonen. 